\title{
Pyometra in a hemodialysis patient with severe constipation
}

\author{
Kohkichi Morimoto ${ }^{1} \cdot$ Tadashi Yoshida $^{1}\left[\right.$ Mototsugu Oya ${ }^{1,2}$ \\ Received: 17 March 2020 / Accepted: 29 April 2020 / Published online: 15 May 2020 \\ (c) Japanese Society of Nephrology 2020
}

Keywords Hemodialysis · Constipation · Phosphate binders

An 87-year-old woman undergoing intermittent hemodialysis suffered from prolonged fever, leukocytosis, and an increase in C-reactive protein. The use of antibiotics was ineffective and she was referred to our hospital. Computed tomography revealed a pooling of fluid with gas within the uterus and a huge amount of stools with metallic density in the colon (Fig. 1). She was diagnosed as having pyometra due to severe constipation. Following the forced defecation by an enema, the pus flowed out from the endometrial cavity, and her conditions improved. Since then, pyometra has not recurred over a year by the control of defecation.

Severe constipation is often seen in hemodialysis patients, because their water intake is restricted and because many of them are prescribed with medications possibly causing constipation, such as phosphate binders. Pyometra is a rare disease condition resulting from cervical occlusion [1].

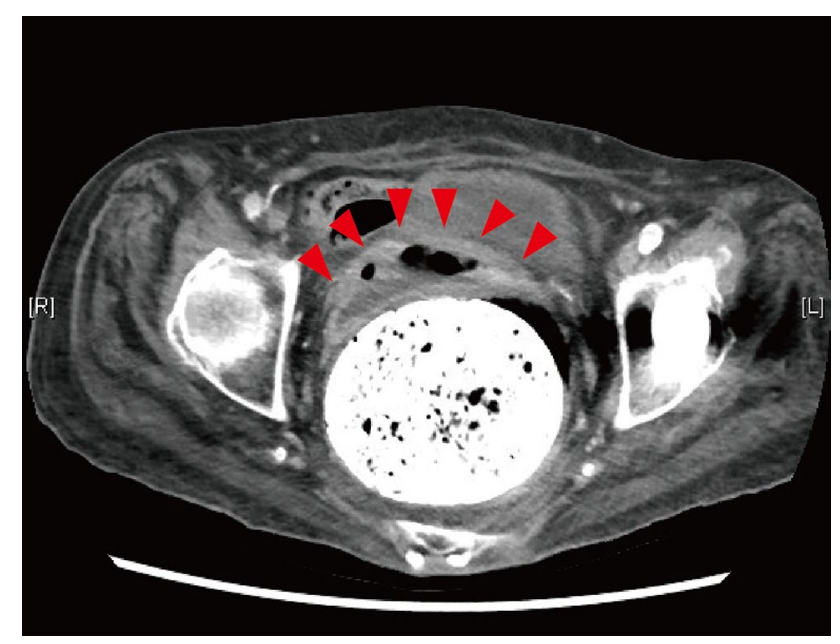

Fig. 1 Abdominal computed tomography showing fluid retention with gas in the uterus (Arrowheads) and a large amount of stools with metallic density in the colon

Physicians should be aware that severe constipation could be a cause of it.

\section{Compliance with ethical standards}

Conflict of interest The authors have declared that no conflict of interest exists.

Human and animal rights This article does not contain any studies with human participants or animals performed by any of the authors.

Tadashi Yoshida

tayoshida-npr@umin.ac.jp

\section{Reference}

1 Apheresis and Dialysis Center, School of Medicine, Keio University, 35 Shinanomachi, Shinjuku-ku, Tokyo 160-8582, Japan

2 Department of Urology, School of Medicine, Keio University, Tokyo, Japan

1. Chan LY, Lau TK, Wong SF, Yuen PM. Pyometra: what is its clinical significance? J Reprod Med. 2001;46:952-6.

Publisher's Note Springer Nature remains neutral with regard to jurisdictional claims in published maps and institutional affiliations. 